University of Nebraska - Lincoln

DigitalCommons@University of Nebraska - Lincoln

Edward Schmidt Publications

Research Papers in Physics and Astronomy

September 1990

\title{
TWO NEW SHORT-PERIOD CEPHEIDS
}

Edward G. Schmidt

University of Nebraska-Lincoln, eschmidt1@unl.edu

Bradley A. Gross

University of Nebraska - Lincoln

Follow this and additional works at: https://digitalcommons.unl.edu/physicsschmidt

Part of the Physics Commons

Schmidt, Edward G. and Gross, Bradley A., "TWO NEW SHORT-PERIOD CEPHEIDS" (1990). Edward Schmidt Publications. 31.

https://digitalcommons.unl.edu/physicsschmidt/31

This Article is brought to you for free and open access by the Research Papers in Physics and Astronomy at DigitalCommons@University of Nebraska - Lincoln. It has been accepted for inclusion in Edward Schmidt Publications by an authorized administrator of DigitalCommons@University of Nebraska - Lincoln. 


\title{
TWO NEW SHORT-PERIOD CEPHEIDS
}

\author{
EDWARD G. SCHMIDT AND BRADLEY A. GROSS
}

Behlen Observatory, Department of Physics and Astronomy, University of Nebraska

Lincoln, Nebraska 68588-0111

Received 1990 May 11

\begin{abstract}
The General Catalogue of Variable Stars gives periods of slightly less than three-quarters of a day for the stars NO Cas and CN Tau. However, new photometry demonstrates that their periods are actually 2.6 and 1.8 days, respectively, and they are thus classical Cepheids. Fourier decompositions of their light curves are performed, and they are found to be members of a class of Cepheids with periods less than three days which may be related to the s-Cepheids. These two stars represent the shortest and longest known members of this class and thus are very useful in defining its properties in the Fourier diagrams.
\end{abstract}

Key words: variable stars-short-period Cepheids-photometry

\section{Introduction}

In the course of the Behlen Observatory variable-star survey (described by Schmidt 1989, 1990) it was found that NO Cassiopeiae and CN Tauri had periods near two days rather than somewhat less than three-quarters of a day as listed in the General Catalogue of Variable Stars (Kholopov 1985, 1987). This, together with their galactic latitudes, indicates that both are short-period classical Cepheids.

Simon and Moffett (1985), Antonello and Poretti (1986), and Moffett and Barnes (1986) have analyzed the light curves of short-period Cepheids using Fourier decomposition. The Fourier parameters show regular sequences for periods longer than a few days, although the so-called s-Cepheids (i.e., those with small amplitude and nearly sinusoidal light curves) form a separate sequence from the others. However, at the shortest periods, the Fourier parameters depart significantly from the sequences. Simon and Moffett suggested that this might be due to overtone pulsation, since two of the stars in question, $\mathrm{SU}$ Cassiopeiae and DT Cygni, have been considered to be overtone pulsators on other grounds (Gieren 1982; Arellano Ferro 1984). Antonello and Poretti, on the other hand, suggested that the short-period stars may be a continuation of the s-Cepheid sequence. If so, these stars may experience a mode resonance near three days.

In view of the uncertainties regarding the shortest period Cepheids, further examples are clearly of interest. For this reason, light curves with sufficient phase coverage for Fourier decomposition have been obtained for NO Cas and CN Tau.

\section{The Observations}

The observations were made with the CCD photome- ter on the 76-cm telescope at Behlen Observatory between September 1988 and January 1990. The intrument and the techniques were described by Schmidt et al. (1990).

Several comparison stars were available in the CCD field for each of the variables. These comparison stars are identified in Table 1 by their $X$ and $Y$ coordinates (in arc seconds, positive to the north and east) relative to the variable. Each field was observed on a number of photometric nights, and mean colors and magnitudes based on those nights are tabulated together with their internal errors and the number of nights used in the mean. In the final determination of differential magnitudes comparison stars C3 and C4 were used for NO Cas and C2, $\mathrm{C} 3$, and $\mathrm{C} 4$ were used for CN Tau.

Light curves are plotted in Figure 1 while Table 2

TABLE 1

Comparison Star Data

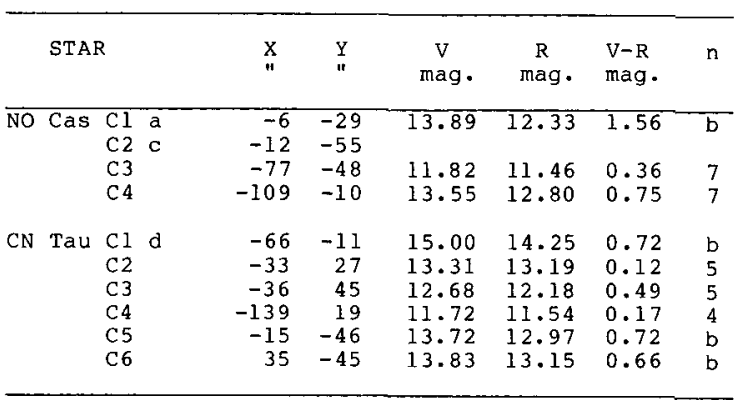

\footnotetext{
Scatter in NO Cas Cl larger than normal. Not used as a comparison star.

Magnitudes and colors determined from other comparison stars differentially.

No photometry was obtained for No Cas C2. It is 1 isted for completeness.

CN Tau Cl has a faint companion. Not used as comparison star.
} 

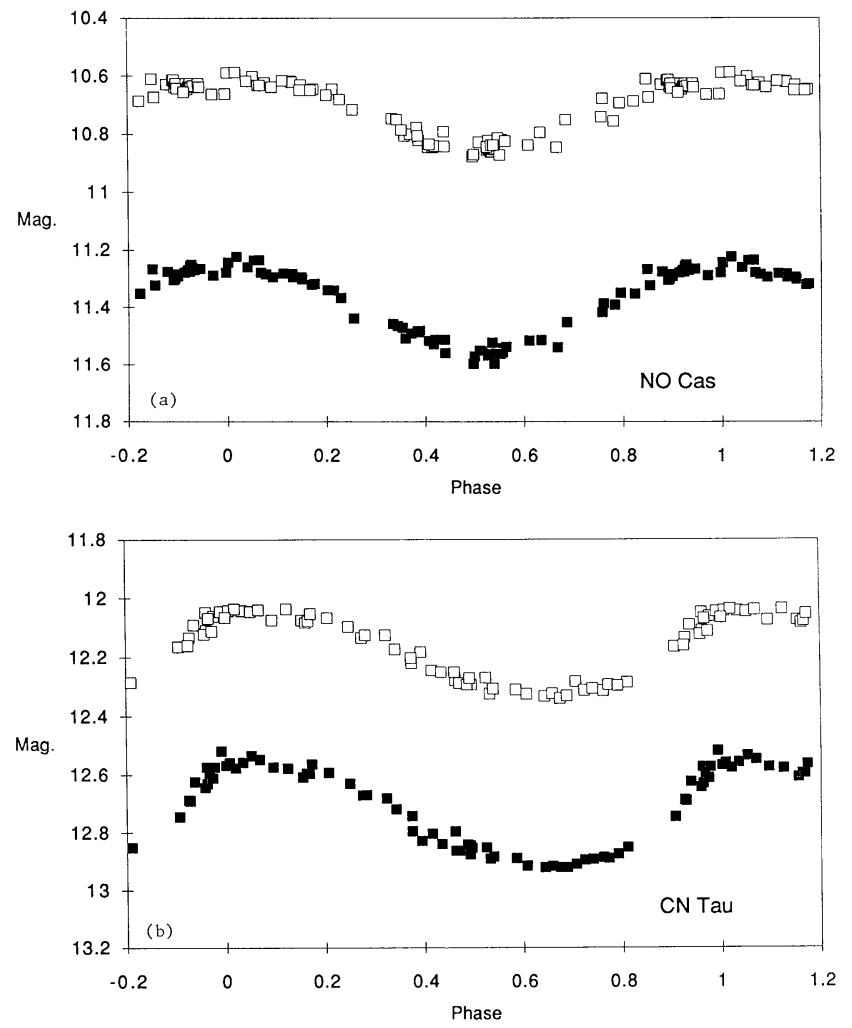

FIG. 1-Light curves in $V$ and $R$. Filled squares represent $V$ magnitudes and open squares the $R$ magnitudes.

TABLE 2

Photometric Data

\begin{tabular}{|c|c|c|}
\hline & NO Cas & CN Tau \\
\hline $\begin{array}{l}\text { Period } \\
\text { Epoch of } \\
\text { Maximum }\end{array}$ & $\begin{array}{c}2.5829+/-0.0002 \\
2,447,771.69\end{array}$ & $\begin{array}{r}1.793+/-0.001 \\
2,447,548.75\end{array}$ \\
\hline $\begin{array}{l}\text { Number of } \\
\text { observations }\end{array}$ & 84 & 61 \\
\hline $\begin{array}{c}\langle\mathrm{V}\rangle \mathrm{a} \\
\langle\mathrm{R}\rangle \mathrm{a} \\
\mathrm{V}(\max ) \\
\mathrm{V}(\min ) \\
\mathrm{R}(\max ) \\
\mathrm{R}(\min )\end{array}$ & $\begin{array}{l}11.41 \\
10.73 \\
11.26 \\
11.58 \\
10.62 \\
10.86\end{array}$ & $\begin{array}{l}12.74 \\
12.20 \\
12.54 \\
12.93 \\
12.04 \\
12.34\end{array}$ \\
\hline $\begin{array}{c}\mathrm{R}(21) \\
\mathrm{Phi}(21)\end{array}$ & $\begin{array}{r}0.085 \\
5.8:\end{array}$ & $\begin{array}{l}0.145 \\
4.35\end{array}$ \\
\hline
\end{tabular}

a Intensity means.

presents various quantities derived from our photometry. The individual light-curve points are available from the first author by electronic mail or by sending a $5^{1 / 2-i n c h ~ P C ~}$ diskette.

The periods were obtained in a two-step process. Approximate values were derived using a data-compensated discrete Fourier transform (Ferraz-Mello 1981) program written by E. P. Belserene. These were then refined by plotting light curves with slightly different periods and seeking the minimum scatter. The errors given in the table represent a change which was large enough to produce significant increases in the scatter and should, thus, be maximum uncertainties in the periods. The light curves are rather flattopped so the dates of maximum are poorly defined. The maximum and minimum magnitudes are the means from points near in phase to the extrema of the light curves, while the mean magnitudes were derived from the Fourier fits discussed below.

\section{Fourier Decomposition}

We have fitted Fourier series to the $V$ and the $R$ light curves. The light curves of both stars are nearly sinusoidal and, thus, the terms beyond the first in the Fourier series are small. In fact, none have amplitudes larger than 0.02 magnitude. This being the case, only second-order fits were needed and no improvement was realized by going to higher orders; the scatter about the fitted curves remained at 0.02 magnitude. However, while increasing the order did not affect the amplitudes of the first and second terms, their phases were less stable than is usual in this type of analysis. Table 2 lists the Fourier parameters in the notation of Simon and Moffett (1985). The $R(21)$ values are simple means between the values derived from the $V$ and $R$ light curves while the phi(21) values from the $R$ light curves were decreased by 0.2 (Simon and Moffett 1985) before averaging. While the $R(21)$ values agree to better than 0.07 between $V$ and $R$, the phase differences (after the correction to the $R$ values) differ by 0.1 for CN Tau and 1.2 for NO Cas. Additionally, the phase differences for NO Cas changed significantly as the order of the fit was increased. Thus, although we give a value for this star, it should be considered very approximate. This is due to the small amplitude of the second term in the Fourier series, less than 0.01 magnitude.

\section{Discussion}

In Figure 2 the amplitude ratio, $R(21)$, and the phase difference, phi(21), are plotted for Cepheids with periods shorter than ten days. Data from Simon and Moffett (1985), Moffett and Barnes (1986), and Antonello and Poretti (1986) are represented by filled squares, while those from Table 2 are shown as open squares.

Looking first at the amplitude ratio diagram (Fig. 2a), we see two distinct sequences of points for periods between three and seven days. The upper sequence represents the sample of Simon and Moffett while the sCepheids added by Antonello and Poretti form the lower sequence. The shorter period stars seem to represent a continuation of the s-Cepheid sequence with an inflection near three days. The two stars studied here help to define this behavior since they constitute the longest- and shortest-period stars in the short-period group.

The phase difference diagram (Fig. 2b) shows behavior similar to the amplitude diagram for periods longer than three days. The short-period group, on the other hand, 

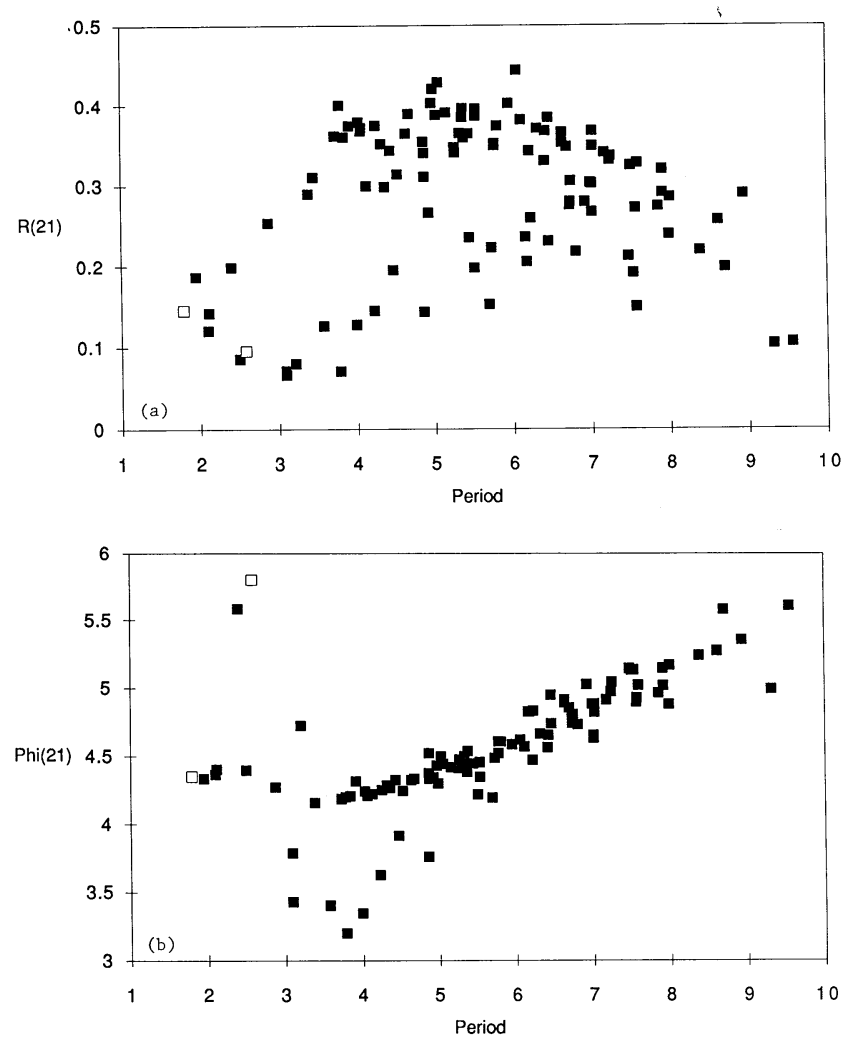

FIG. 2-Fourier parameters for short-period Cepheids. NO Cas and CN Tau are represented by open squares.

falls above the other two sequences and is not obviously connected with the s-Cepheid sequence. However, if we accept the suggestion of Antonello and Poretti that the s-Cepheids experience a resonance at about three days, the sudden jump in phi $(21)$ is similar to the behavior of the Cepheid sequence near the ten-day resonance (see Fig. 2 of Moffett and Simon). Again the two stars of the present study serve to define the sequence better for the shorter period stars by defining the long- and short-period ends. In particular, the appearance of NO Cas at a large value of phi(21) places it near AU Pegasi and suggests that this latter star is simply at an extreme location but is not otherwise abnormal.

We conclude that the addition of two further stars to the short-period Cepheid group has served to support the association of these stars with the s-Cepheids. Obviously, more stars will help to clarify this point further.

The CCD camera system used in this research was obtained through grant AST 85-04072 from the National Science Foundation, and the observations were completed with support from NSF grant AST 88-15806. Data reduction and analysis were performed using the facilities of the Minnich Astronomical Computing Center donated by Commander C. B. Minnich. We thank Dr. E. P. Belserene for the use of her period-search routine.

\section{REFERENCES}

Antonello, E., and Poretti, E. 1986, Astr. Ap., 169, 149.

Arellano Ferro, A. 1984, M.N.R.A.S., 209, 481.

Ferraz-Mello, S. 1981, A.J., 86, 619.

Gieren, W. 1982, Pub. A.S.P., 94, 960.

Kholopov, P. N. 1985, General Catalogue of Variable Stars, Fourth Edition, Vols. 1 and 2 (Moscow: Nauka Publishing House). 1987, General Catalogue of Variable Stars, Fourth Edition, Vol. 3 (Moscow: Nauka Publishing House).

Moffett, T. J., and Barnes, T. G. 1986, M.N.R.A.S., 219, 45P.

Schmidt, E. G. 1989, in Automatic Small Telescopes, ed. D. S. Hayes and R. M. Genet (Mesa, AZ: Fairborn Press), p. 195. 1990, in CCD's in Astronomy II. New Methods and Applications of CCD Technology, ed. A. G. D. Philip and D. S. Hayes (Schenectady, NY: L. Davis Press), in press.

Schmidt, E. G., Loomis, C., Groebner, A. T., and Potter, C. T. 1990, Ap. $J$., in press.

Simon, N. R., and Moffett, T. J. 1985, Pub. A.S.P., 97, 1078. 